\title{
Estimating the solar wind pressure at comet 67P from Rosetta magnetic field measurements
}

\author{
Aniko Timar ${ }^{1,2^{*}}$, Zoltan Nemeth ${ }^{1}$, Karoly Szego ${ }^{1}$, Melinda Dósa ${ }^{1}$, Andrea Opitz ${ }^{1}$, and Hadi Madanian ${ }^{3}$ \\ 1 Wigner Research Centre for Physics, Institute for Particle and Nuclear Physics, Konkoly-Thege M. Rd. 29-33, 1121 Budapest, Hungary \\ 2 Eötvös Loránd University, Faculty of Science, Pázmány Péter stny. 1, 1117 Budapest, Hungary \\ 3 University of Kansas, Department of Physics and Astronomy, 1082 Malott Hall, 1251 Wescoe Dr, Lawrence, KS 66045, USA
}

Received 13 July 2018 / Accepted 17 December 2018

\begin{abstract}
Aims: The solar wind pressure is an important parameter of space weather, which plays a crucial role in the interaction of the solar wind with the planetary plasma environment. Here we investigate the possibility of determining a solar wind pressure proxy from Rosetta magnetic field data, measured deep inside the induced magnetosphere of comet $67 \mathrm{P} /$ Churyumov-Gerasimenko. This pressure proxy would be useful not only for other Rosetta related studies but could also serve as a new, independent input database for space weather propagation to other locations in the Solar System.

Method: For the induced magnetospheres of comets the magnetic pressure in the innermost part of the pile-up region is balanced by the solar wind dynamic pressure. Recent investigations of Rosetta data have revealed that the maximum magnetic field in the pile-up region can be approximated by magnetic field measurements performed in the inner regions of the cometary magnetosphere, close to the boundary of the diamagnetic cavity, from which the external solar wind pressure can be estimated.

Results: We were able to determine a solar wind pressure proxy for the time interval when the Rosetta spacecraft was located near the diamagnetic cavity boundary, between late April 2015 and January 2016. We then compared our Rosetta pressure proxy to solar wind pressure extrapolated to comet $67 \mathrm{P}$ from near-Earth. After the exclusion of disturbances caused by transient events, we found a strong correlation between the two datasets.
\end{abstract}

Keywords: solar wind / unmagnetized body / magnetic field / space weather / pressure

\section{Introduction}

Solar wind dynamic pressure is the most important external condition, which determines the shape and size of a magnetosphere, and also influences several other magnetospheric boundaries and currents. Both intrinsic and induced magnetospheres compress and spread out depending on the strength of the upstream solar wind pressure (Sibeck et al., 1991). It has been reported that solar wind pressure changes can heavily affect auroral processes at Earth (Boudouridis et al., 2003) and Saturn (Crary et al., 2005), and lightly in the case of Jupiter (Clarke et al., 2009). High solar wind pressure events can also cause the large-scale magnetization of the ionosphere of Venus (Luhmann et al., 1980). The layer around Mars practically void

\footnotetext{
${ }^{*}$ Corresponding author: timar.aniko@wigner.mta.hu
}

of solar wind plasma called the magnetospheric cavity (inside the ion composition boundary), varies from 4500 to $9500 \mathrm{~km}$ in size depending on the strength of the solar wind pressure, and can also virtually disappear at anomalously high external pressure values (Dubinin et al., 1996). At comets, the extent of the diamagnetic cavity is highly sensitive to the variations of the solar wind pressure (Timar et al., 2017).

Thus, many magnetospheric investigations would benefit from knowing the solar wind pressure. The most effective way to get information about solar wind properties is to measure them in situ by spacecraft. This is usually achieved either by solar wind monitors orbiting around the Sun, or in the L1 Lagrange point of Earth, which can even provide solar wind conditions in real-time. Spacecraft targeting planetary bodies can also measure the necessary data while cruising in the solar wind flow before reaching their destination. Spacecraft without dedicated solar wind instruments or deep inside a magnetosphere of a planetary body or a comet (such as Rosetta) 
however, cannot measure it directly. Solar wind monitoring spacecraft are sometimes millions of kilometres away from the point of our interest, in which case researchers have to rely on solar wind data extrapolated by various propagation tools. These tools often have certain limitations, beyond which they cannot provide reliable predictions.

The magnetospheric structures of celestial objects without global magnetic fields are commonly controlled by the interaction with the Sun. For an object with an atmosphere, an ionosphere will be formed in the upper part of the atmosphere from the ionisation of atmospheric atoms and molecules by solar EUV radiation. Since the solar plasma controls the dynamics of the solar wind, the interplanetary magnetic field will be forced to follow the solar wind particles around the obstacle. The convecting magnetic field generates currents in the ionosphere that creates a canceling field, preventing the field to reach the lower parts of the atmosphere. This interaction causes the interplanetary magnetic field to pile up and drape itself around the object. By measuring the properties of the magnetic field deep inside an induced magnetosphere it is possible to estimate the solar wind pressure around planetary bodies by assuming that the magnetic field pressure in the pile-up region balances the solar wind dynamic pressure. This was done by Crider et al. (2003), who developed a method to deduce the solar wind pressure at the position of Mars using the magnetic field measurements of the magnetometer on the Mars Global Surveyor (MGS) spacecraft. It should be noted that Mars still possesses remnant intrinsic magnetic field in some regions which complicate the deduction of a solar wind proxy from orbital spacecraft data.

However, induced cometary magnetospheres (where the solar wind flow is disturbed due to the presence of the comet) differ in many ways from induced planetary magnetospheres, therefore we cannot directly apply Crider's method to, for example, comet 67P/Churyumov-Gerasimenko (67P). Unlike Mars, comets are completely non-magnetic bodies (Auster et al., 2015). Planets and comets also differ in size and mass which affects the processes taking place in their magnetospheres. Moreover, the relationship of the solar wind dynamic pressure and the measured magnetic field also depends on the trajectories of the observing spacecraft or even on the activity of the Sun and the comet at the time of the measurements.

In case of comets, the gravitational pull is so small due to the low mass of these objects that their volatiles heated up by the radiation of the Sun can escape from the nucleus. This neutral gas outflow coming from the comet at high speeds is continuously ionised by EUV photoionisation (Mendis et al., 1985; Cravens, 1991; Madanian et al., 2016a) and collisional ionisation (Gan and Cravens 1990; Wedlund et al., 2017); thus there is a mix of neutral and ionised particles escaping from the nucleus. If the speed of the plasma and the neutral gas differs for some reason, an ion-neutral friction force will arise between these two species (Cravens, 1986; Ip and Axford, 1987).

After Giotto's journey to comet Halley, the neutral-drag model was established (Cravens, 1986; Ip and Axford, 1987) to explain the presence of the diamagnetic cavity, a region around the nucleus defined by its lack of solar wind magnetic field; the ion-neutral drag force acting on the ionised particles by the rapidly escaping neutral flow in a cometary atmosphere is able to withstand the solar wind magnetic pressure:

$$
\nabla \frac{B^{2}}{2 \mu_{0}}=m_{\mathrm{i}} n_{\mathrm{i}} v_{\text {in }}\left(u_{n}-u_{\mathrm{i}}\right)
$$

where $B$ is the magnetic field, $n_{i}, m_{i}$ and $u_{i}$ are the number density, mass and velocity of cometary ions, $u_{n}$ is the neutral velocity and $v_{\text {in }}$ is the ion-neutral collision coefficient. The term on the left-hand side is the magnetic pressure gradient which equals the ion-neutral drag force on the right-hand side, under the assumption that the magnetic tension, dynamic pressure and thermal pressures are negligible (for a full derivation, see Cravens, 1986). The magnetic field lines are slowed down and redirected in front of this obstacle created by the ion-neutral drag and cannot penetrate further towards the nucleus, creating a diamagnetic cavity. Arriving to the diamagnetic cavity boundary, the ionised particles escaping from the nucleus are trapped by the magnetic field; the ions are halted and the approximation $u_{i} \approx 0$ can be used at the boundary (Cravens, 1987). The diamagnetic cavity boundary (also called the contact surface or cavity surface) is the innermost boundary of a cometary atmosphere, separating its magnetized and non-magnetized regions. The extent of the cavity was measured to be $4470 \mathrm{~km}$ (inbound) and $4155 \mathrm{~km}$ (outbound) during Giotto's encounter with comet Halley (Neubauer et al., 1986). In case of comet 67P the extent of the cavity is much smaller due to the comet's lower production rate, around $50-400 \mathrm{~km}$.

Upstream of the diamagnetic cavity the solar wind magnetic field lines bend and pile up, creating the induced magnetosphere of the comet. The dynamic pressure of the solar wind compresses the induced cometary magnetosphere; the magnetic pressure of the compressed magnetic field balances the pressure of the incoming solar wind $\left(p_{\mathrm{sw}}\right)$ :

$$
p_{\mathrm{sw}}=\frac{B_{0}^{2}}{2 \mu_{0}}
$$

where $B_{0}$ is the maximum of the magnetic field magnitude in the magnetic pile-up region, where the magnetic field is the most compressed. A detailed description of cometary plasma regions and boundaries can be found in Mandt et al. (2016).

When the solar wind dynamic pressure increases, the induced magnetosphere is compressed until the increasing magnetic pressure of the compressed field is once again able to withstand the dynamic pressure of the solar wind. Thus by estimating $B_{0}$, we can estimate the solar wind dynamic pressure as well.

The Rosetta spacecraft (Glassmeier et al., 2007a) was launched in March 2004 and arrived to comet 67P in August 2014. Rosetta accompanied 67P for a 2-year long period through the comet's perihelion on 13 August 2015 while making observations of the cometary environment. The Rosetta mission concluded on 30 September 2016 when the spacecraft hard landed on the cometary surface.

In this paper we describe a method, which can be used to derive an in situ solar wind pressure proxy for the location of comet 67P based on Rosetta magnetic field measurements (Glassmeier et al., 2007b; Glassmeier, 2017; Goetz et al., 2017), for such times when Rosetta was deep inside the cometary magnetosphere, near the diamagnetic cavity boundary. This time period is between late April 2015 and January 2016. We then compare our pressure proxy data to extrapolated 
solar wind pressure measured by near-Earth spacecraft. We also test the accuracy of this pressure proxy on other phenomena observed in the plasma environment of comet 67P, which are expected to be also sensitive to the variation of the solar wind pressure. For this, we assume a global diamagnetic cavity around comet $67 \mathrm{P}$, where the cavity boundary distance changes in response to the changes in the solar wind dynamic pressure, and in the global outgassing rate of the comet.

\section{Comparison of $1 \mathrm{P} /$ Halley and 67P}

One can compare the neutral and ion densities and velocities measured and modelled at comets Halley and 67P to see if the conditions necessary to apply the model are satisfied for $67 \mathrm{P}$ as well, which is a comet with much lower activity than Halley.

Although comets Halley and 67P differ very much in nucleus size and activity, the regions where the diamagnetic cavity is formed are very similar in neutral and ion density values for the two comets. In case of Halley, the cavity is located where the neutral density is around $5 \times 10^{6} \mathrm{~cm}^{-3}$ (using $n_{n}=Q / 4 \pi u_{n} r^{2}$ for the neutral density, where $Q=9.3 \times 10^{29} \mathrm{~s}^{-1}$ is the outgassing rate of comet Halley and $r=4100 \mathrm{~km}$ is the cometocentric distance for the outbound cavity boundary; Cravens, 1986). In order to calculate the neutral density profile at Halley, a constant neutral velocity of $u_{n} \approx 1 \mathrm{~km} \mathrm{~s}^{-1}$ was used. At comet $67 \mathrm{P}$, the neutral density measured by the ROSINA instrument (Balsiger et al., 2007) is around $1-2 \times 10^{7} \mathrm{~cm}^{-3}$ in early July 2015 and an overall $r^{-2}$ fall off of the neutral densities were also reported (Bieler et al., 2015; Hässig et al., 2015).

At comet Halley, Cravens (1987) investigated the ion density dependence on the cometocentric distance. An ion species is photochemically controlled by recombination processes if its chemical lifetime is much less than its transport time. At Halley, Cravens (1987) reported ion chemical lifetimes of $\sim 10 \mathrm{~s}$ (for $n_{n} \approx 10^{7} \mathrm{~cm}^{-3}$ ) and transport time of about $1000 \mathrm{~s}$ for $r=1000 \mathrm{~km}$ (using $u_{n} \approx 1 \mathrm{~km} \mathrm{~s}^{-1}$ ), therefore the ions are generally photochemically controlled. Cravens (1987) calculated the density profiles using photochemical equilibrium and found that the ion density varies inversely with the cometocentric distance $\left(r^{-1}\right)$. He also calculated the ion density function assuming only loss by transport and neglecting recombination altogether, in which case they also found an $r^{-1}$ dependence but concluded that photochemical equilibrium is a better approximation for the ion density profile than transport.

At comet 67P, near the diamagnetic cavity boundary, since the densities are similar $\left(n_{n} \approx 10^{7} \mathrm{~cm}^{-3}\right)$, chemical lifetimes should be comparable to those at Halley $(\sim 10 \mathrm{~s})$, however here we are at $150-300 \mathrm{~km}$ from the comet, so the transport time is only hundreds of seconds. This still indicates a photochemically controlled regime, although transport could also be somewhat relevant. Edberg et al. (2015) reported that the ion density falls off with radial distance with approximately $r^{-1}$ in early 2015 within $260 \mathrm{~km}$ from the nucleus of 67P, based on Rosetta measurements. Galand et al. (2016) and Vigren et al. (2016) also investigated the ion densities at $67 \mathrm{P}$ at relatively large heliocentric distances $(>2.5 \mathrm{AU})$ and agreed to the $r^{-1}$ dependence also observed at Halley. Both photochemical equilibrium and transport dominant models give the same qualitative spatial dependence for the ion density at both comets. Since the Cravens model only required the $r^{-1}$ fall-off of the ion density, the equations of Cravens (1987) should work here as well, up to a constant of the order of 1.

Vigren and Eriksson (2017) reported ambipolar electric fields in the coma of 67P near perihelion (August, 2015) that can possibly accelerate ions up to $8 \mathrm{~km} \mathrm{~s}^{-1}$, higher than the neutral velocities inside the diamagnetic cavity of $67 \mathrm{P}$. This would mean that in the cavity, the direction of the neutral drag force points inward. But upon reaching the cavity boundary, the cometary ions are abruptly halted, and we can use the approximation $u_{i} \approx 0$ in the boundary layer (Cravens, 1986), allowing the neutral flow to act on the ions to withstand the external magnetic pressure.

\section{Data}

To calculate our Rosetta magnetic field pressure proxy, we use the measurements of the Rosetta Plasma Consortium magnetometer (RPC MAG) (Glassmeier et al., 2007b) and the Rosetta Orbiter Spectrometer for Ion and Neutral Analysis (ROSINA) (Balsiger et al., 2007).

The RPC MAG is made up of two (inboard and outboard) triaxial fluxgate magnetometer mounted on a $1.5 \mathrm{~m}$ boom separated from each other by $15 \mathrm{~cm}$ and measures the magnetic field vector in the range $\pm 16384 \mathrm{nT}$ with quantization steps of $31 \mathrm{pT}$ and a time resolution up to $0.05 \mathrm{~s}$ (Glassmeier et al., 2007b).

The ROSINA consist of two mass spectrometers for neutral and ion analysis and two pressure gauges (Comet Pressure Sensor [COPS]) that provide density and velocity measurements of the cometary gas (Balsiger et al., 2007). In this paper we use the time dependent, global water production rate calculated from the local neutral density measurements of ROSINA by Hansen et al. (2016). They corrected the local density measurements for spacecraft motion, radial distance from the comet, Sun-fixed longitude and latitude, then averaged over a cometary rotation period to get a global outgassing rate.

We compared our Rosetta pressure proxy to solar wind dynamic pressure measurements of different spacecraft at various locations in the solar system, extrapolated to the location of the comet using three different propagation methods. The first propagation technique is the simple ballistic method which assumes that solar wind bulk velocity is constant during radial propagation (Vennerstrom et al., 2003; Opitz et al., 2009). The second method is the magnetic lasso model, which is an enhanced ballistic method based on the reconstruction of the magnetic connectivity between the solar source and the target (Dósa et al., 2018). We also used the extrapolated solar wind dynamic pressure data of the mSWiM model which is based on 1D MHD for the radial propagation while using ballistic propagation for the solar rotation (Zieger and Hansen, 2008).

All three methods were applied to the OMNI solar wind pressure generated using the combined solar wind measurements of near-Earth spacecraft, while the ACE dataset was also extrapolated from near-Earth to the position of the comet with the ballistic and lasso models. Comet $67 \mathrm{P}$ was relatively far away from Earth $\left(\sim 80-140^{\circ}\right)$ in this time period (Fig. 1), which required the extrapolation of the near-Earth solar wind pressure 
data by approximately 10 days in April 2015 and 4-5 days in January 2016. This great distance decreases the accuracy of the extrapolation, especially during intense solar activity and low solar source persistence.

Early to mid 2015 the comet was located relatively near the STEREO-A spacecraft $\left(\sim 45-80^{\circ}\right)$, but unfortunately, STEREOA measurements were mainly available in late 2015, at which time the comet was again closer to the near-Earth solar wind monitoring spacecraft (Fig. 1). Still, we propagated the sparsely available STEREO-A data to the position of comet 67P using the ballistic method.

\section{Method}

The magnetic pressure $\left(B_{0}^{2} / 2 \mu_{0}\right)$ would be a good proxy to estimate the solar wind dynamic pressure around comet 67P, however, it is usually difficult to determine the $B_{0}$ maximum of the pile-up region, mainly because of the rapidly changing solar wind pressure around the comet, and the varying orbital trajectory of Rosetta. In order to directly measure the $B_{0}$ maximum, a spacecraft should always be at the location, where the field is most compressed, which is a very specific and swiftly changing spatial position inside the magnetosphere. Therefore, instead of direct measurements of $B_{0}$, we have to rely on some known relationships, which connect $B_{0}$ with the magnetic field amplitude measured at the position of the spacecraft $(B(r))$. Since the magnetosphere is a complex and dynamic environment, it is not always possible to find such a relationship, especially a relationship accurate enough to be useful as a basis of a pressure proxy.

After the Giotto probe passed through the diamagnetic cavity of comet Halley, several models were created to describe how the strength of the magnetic field changes depending on the distance from the nucleus and the $B_{0}$ field maximum (Cravens, 1986, 1987; Wu, 1987; Ip and Axford, 1987; Galeev, 1986). In this paper we follow the solution of the neutral-drag model calculated by Cravens $(1986,1987)$ that determines the shape of the $B(r)$ function for magnetic field values measured between the diamagnetic cavity boundary and infinity, in which the magnetic field monotonically grows from zero to its maximum $\left(B_{0}\right)$ with increasing cometary distance:

$$
B(r)=B_{0} \sqrt{1-\frac{r_{\mathrm{cs}}^{2}}{r^{2}}}
$$

where $r_{\mathrm{cs}}$ is the diamagnetic cavity boundary distance: $r_{\mathrm{cs}}=c \cdot \frac{Q^{3 / 4}}{B_{0}}, c=7.08 \times 10^{-18}\left[\mathrm{~km} \mathrm{nT} \mathrm{s}^{3 / 4}\right]$ is a constant (Cravens, 1986; Madanian et al., 2016b).

The solution, of course, is only valid, where the gradient of the magnetic pressure dominates the stress due to field line curvature. This is true near the cavity, where the field changes rapidly with $r$, thus the gradient is high. The field approaches its theoretical maximum quickly, a diminishing gradient means that the field is already close to $B_{0}$, thus we can approximate the maximum from the value of the Cravens solution at a finite distance (Fig. 2). In the vicinity of the diamagnetic cavity the neutral-drag dominates the physical processes, and the model is able to predict not only the size of the cavity $\left(r_{\mathrm{cs}}\right)$, but also the field magnitude as a function of $B_{0}$ and $r$. We have inverted this $B\left(B_{0}, r\right)$ relationship to get the desired $B_{0}$ values from the measurements. After some calculations we can get:

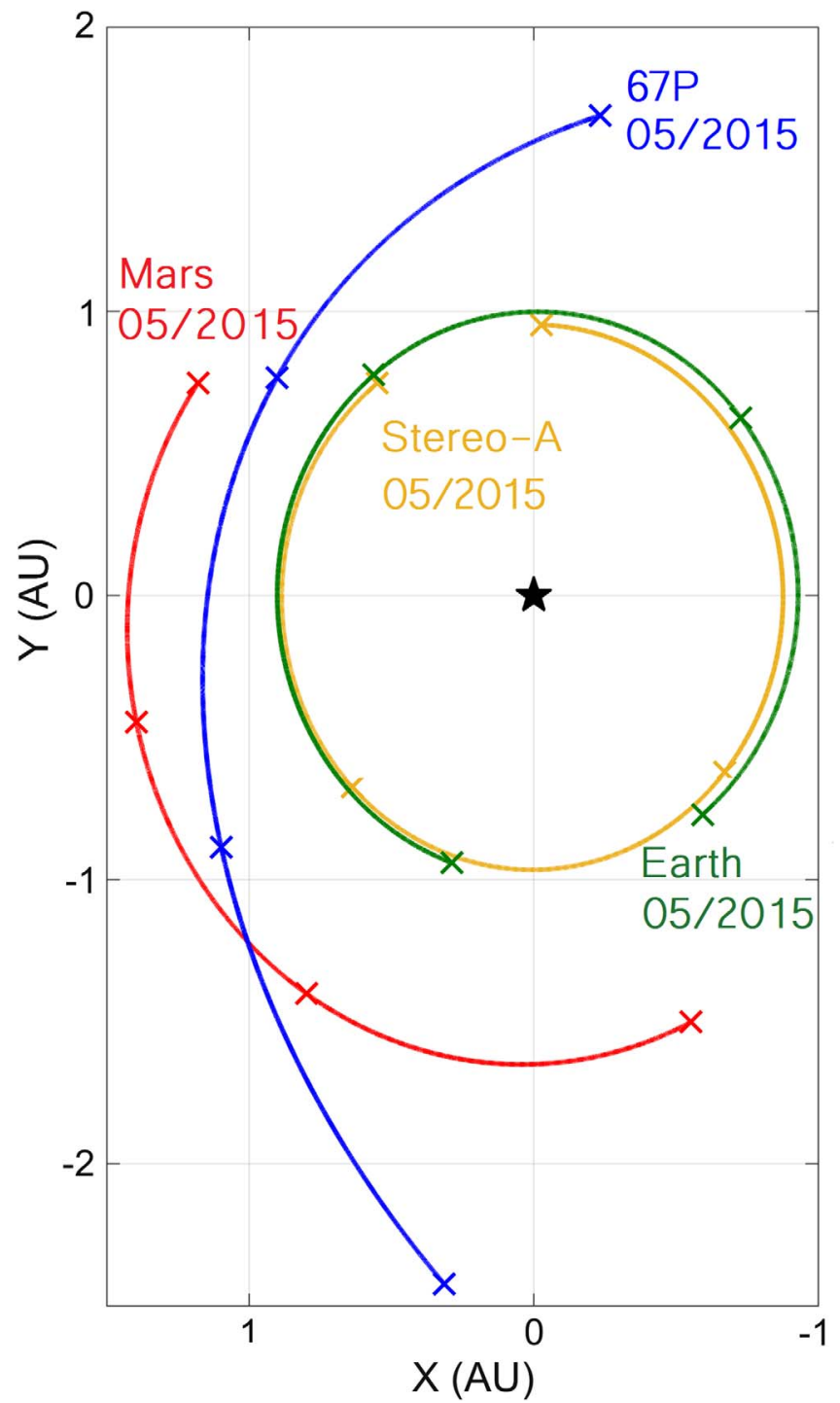

Fig. 1. Positions in the solar system in J2000 coordinate system between May 2015 and March 2016. The Sun is in the origin, comet 67P's trajectory is illustrated with a blue, the Earth's with a green, Mars' with a red and the STEREO-A spacecraft's with a yellow line. The coloured x's mark the same dates on each line: the first $\mathrm{x}$ marks 1 May 2015, the second 1 August 2015, the third 1 November 2015 and the last one marks 1 March 2016.

$$
B_{0}^{2}=B(r)^{2}+c^{2} \frac{Q^{3 / 2}}{r^{2}} .
$$

Using equation (4) we can calculate $B_{0}$, and by combining it with equation (2), the solar wind pressure can also be approximated. The validity of this estimation method is limited to the region, where the Cravens model describes $B(r)$ well - principally the vicinity of the diamagnetic cavity $\left(r \approx 2-3 r_{\mathrm{cs}}\right)$. This means that we can approximate the solar wind dynamic pressure from magnetic field data measured near the cavity.

It is also possible to apply a somewhat more general approach. If we can estimate $B_{0}$ without any specific knowledge about the exact shape of the $B(r)$ curve, then it is also possible to find the solar wind dynamic pressure. Suppose that the 

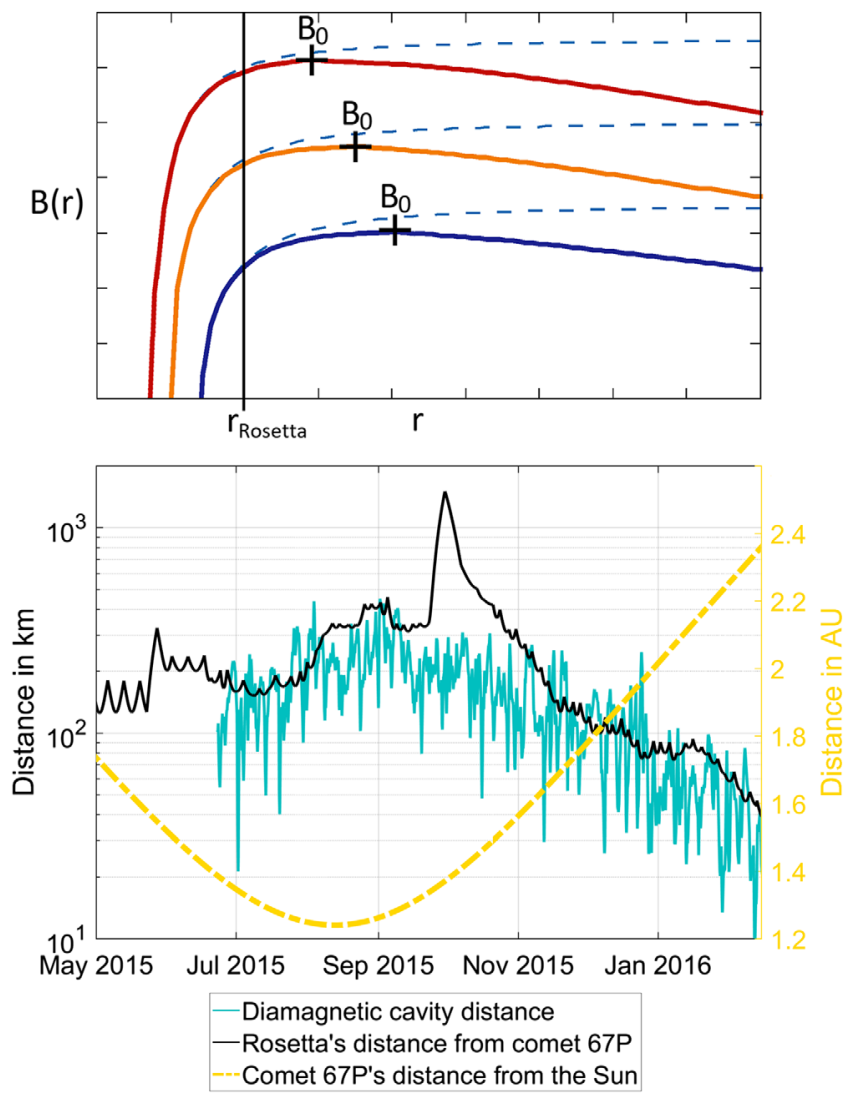

Fig. 2. In the upper panel we illustrate the shape of the $B(r)$ function according to Cravens (dashed blue lines) depending on the cometary distance in different solar wind conditions. The nucleus and the diamagnetic cavity is on the left, the solar wind arrives from the right-hand side. The vertical black line illustrates the distance of the Rosetta spacecraft ( $r_{\text {Rosetta }}$ ) from comet 67P. Monotonically growing from zero to maximum, the Cravens solution reaches the $B_{0}$ value at infinity. In practice, however, the magnetic field reaches its maximum in a finite distance from the diamagnetic cavity boundary then it starts to decrease. The coloured (red, orange, blue) lines illustrate this behaviour. The blue line represents a scenario with relatively low solar wind pressure; the $B_{0}$ maximum (marked by black cross) is far away from both the comet and the location of the spacecraft. When the solar wind pressure increases, the $B_{0}$ maximum is pushed back towards the comet while increasing in value (orange and red lines). The lower panel shows comet 67P's distance from the sun (dashed yellow line), Rosetta's distance from the comet (black line). The diamagnetic cavity's extent calculated using OMNI mSWiM solar wind dynamic pressure is illustrated by the light blue line. The Cravens solution is close to these representations in the upper panel at small cometary distances $\left(r \approx 3 r_{\mathrm{cs}}\right)$.

$B(r)$ curve has only a single peak (the maximum value in the pile-up region), and the solar wind dynamic pressure varies significantly - in such a case the estimation is possible. When the solar wind dynamic pressure increases around the induced cometary magnetosphere, the magnitude of the maximum magnetic field in the pile-up region will also increase, its position being pushed back towards the comet together with the diamagnetic cavity boundary until the ion-neutral drag force inside the

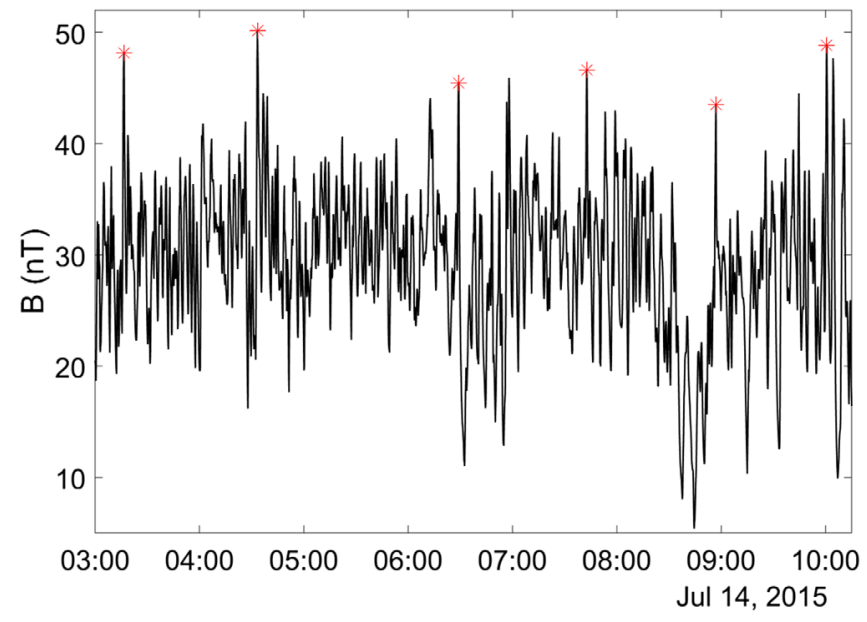

Fig. 3. Rosetta magnetic field data with a temporal resolution of $10 \mathrm{~s}$ on 14 July 2015 . The red asterisks mark the selected peaks if the minimum peak value is $20 \mathrm{nT}$, the minimum peak prominence is $15 \mathrm{nT}$ and the minimum peak distance is $1 \mathrm{~h}$.

cavity builds up to balance the external pressure force. On the other hand, when the solar wind dynamic pressure decreases, the diamagnetic cavity boundary and the location of the maximum of the pile-up region extends outwards, and the $B_{0}$ value is decreasing again. Therefore, in variable solar wind dynamic pressure conditions, both the magnitude and the position of the maximum magnetic field in the pile-up region change. When the solar wind pressure changes upstream, the strength of the magnetic field around the spacecraft located in the induced cometary magnetosphere varies with it. Strong enough variation means that the location of the maximum is sometimes pushed close to the spacecraft (see Fig. 2). Thus, the maxima appearing in the time series data can represent an indicator for the spatial maximum of the field.

This way we can approximate the solar wind pressure by selecting the local maxima of the magnetic field measured by Rosetta to estimate the $B_{0}$ maximum, while assuming that the field changes with the varying solar wind pressure, with no other processes influencing significantly the strength of the magnetic field near the cavity boundary. Our interpretation is that in the vicinity of the diamagnetic cavity, the variations of the magnetic field are mostly caused by the rapidly changing solar wind dynamic pressure as it compresses and dilates the diamagnetic cavity. It is possible that waves propagating along the boundary also contribute to the variation of the magnetic field (Richter et al., 2015, Hajra et al., 2018). Here we assume that the most prominent peaks are caused by solar wind pressure variations and check the validity of our assumptions by comparing our model predictions with measurements. We call this the method of peak-selection (Madanian et al., 2016b). In order to get a reliable estimation, care must be taken to select only the most prominent peaks in a time interval. Smaller peaks can represent waves or small amplitude pressure variations, which cannot push the maximum close to the spacecraft position, thus these are neglected. One can test the reliability of this method by comparing its results with that of the previous method, or to propagated pressure values.

The shape of the $B(r)$ function according to the Cravens model are illustrated for different solar wind conditions in 


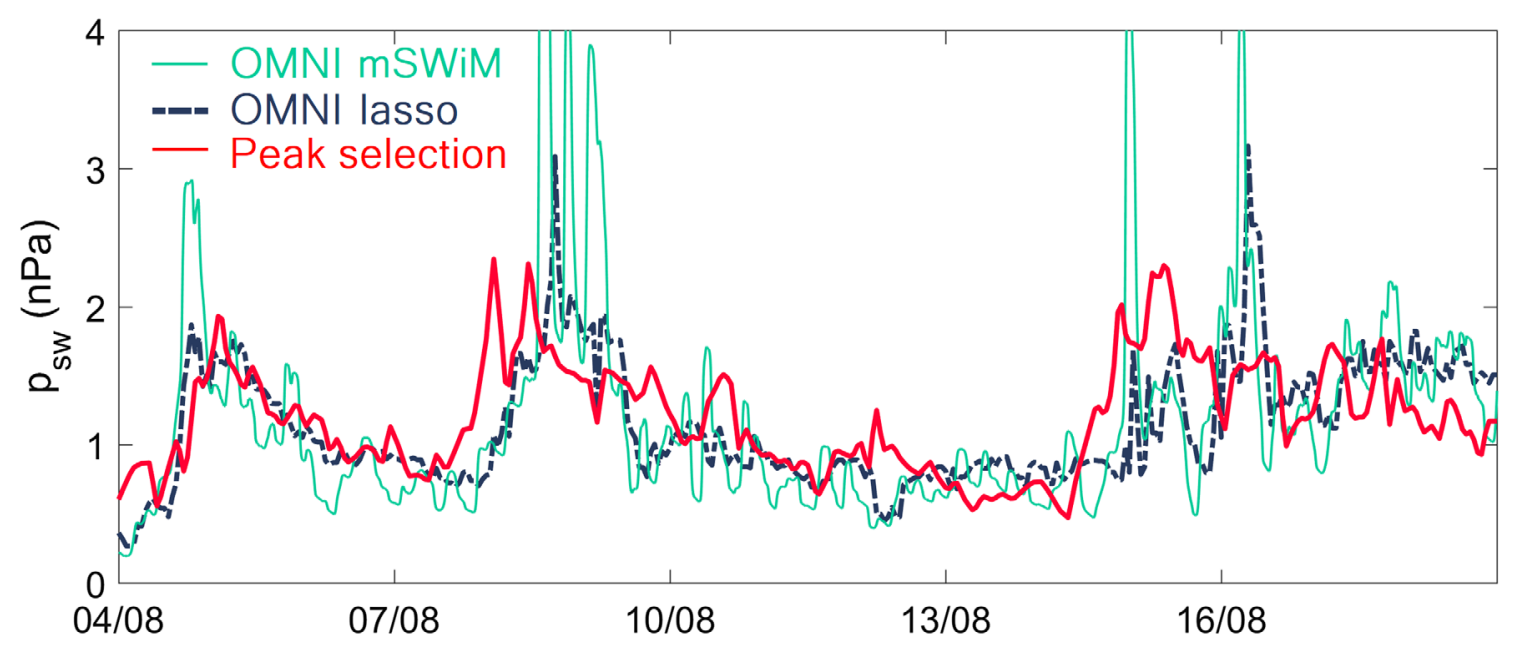

Fig. 4. Rosetta pressure proxy compared to the OMNI lasso and OMNI mSWiM propagated solar wind pressure between 4-19 August 2015. There are three CIRs that reached comet 67P uninterrupted after passing the solar wind monitors near-Earth. With no other transient solar activity, the Rosetta pressure proxy shows great similarity to the propagated datasets.

Figure 2 (dashed lines). Suppose that Rosetta is located inside the position of the maximum value (between the $B_{0}$ maximum value and the comet); if the solar wind pressure is relatively low, the spacecraft will measure weak magnetic field. If the solar wind pressure increases, the $B_{0}$ maximum is pushed back towards the comet while increasing in value; then the spacecraft measures higher magnetic field values, closer to the $B_{0}$ maximum. With the peak selection method, we search for these local peaks in the magnetic field data to estimate the $B_{0}$ maximum. If the spacecraft is located well outside the position of the $B_{0}$ maximum, we are far away from the diamagnetic cavity, the shape of the $B(r)$ function cannot be approximated by the simplest Cravens solution. Still, a pressure variation in this region would also lead to peak magnetic field values close to $B_{0}$. Thus the peak selection method can provide reliable results in a spatial region several times larger than the cavity itself.

We calculated our pressure proxy in the time period when diamagnetic cavity crossings were observed (Goetz et al., 2016; Nemeth et al., 2016), and therefore the approximations of the neutral-drag model can be presumed to be valid: between late April 2015 and January 2016. At that time, the Rosetta spacecraft was orbiting mostly in the terminator plane of the comet.

We assumed that the most prominent peaks observed in the field magnitude are caused by solar wind pressure variations, and that the $B(r)$ function has a maximum $\left(B_{0}\right)$ in the pile-up region. From that we can approximate the solar wind pressure using the peak values as a proxy for $B_{0}$. If we further assume that $B(r)$ follows the Cravens solution, we can compute $B_{0}$ from $B(r)$ without any assumptions about the peak values. The two methods give very similar results, suggesting that the peak selection method finds peak values close to $B_{0}$ and that the Cravens model describes the shape of the $B(r)$ curve fairly well (Fig. 5).

\section{Validation}

We calculated our pressure proxy from the Rosetta magnetic field measurements using both the Cravens solution for $B(r)$ (Eqs. (3) and (4)) and the method of peak selection. The two techniques give very similar results. The calculated pressure of the Cravens solution based method has the same temporal resolution as the input magnetic field data, while the result of the peak-selection has lower resolution due to only using selected local maxima of the input data. The input magnetic field data has a temporal resolution of $10 \mathrm{~s}$ (averaged from data with a resolution of $1 \mathrm{~s}$ ). We choose peaks with a minimum peak value of $20 \mathrm{nT}$, a minimum peak prominence of $15 \mathrm{nT}$ and a minimum peak distance of $1 \mathrm{~h}$ (Fig. 3). When compared, the pressure results from the two methods match each other well (Figs. 5 and 7a). The Cravens solution heavily underestimates the solar wind pressure when the Rosetta spacecraft is located further away from the cavity boundary, most notably in October, 2015. Here, the peak-selection should also give somewhat lower estimates than the actual pressure values. The two methods show the best correlation and are the most accurate when the spacecraft is the closest to the $B_{0}$ maximum of the pile-up region $\left(r \approx 2-3 r_{\mathrm{cs}}\right)$. Here the Cravens solution works well, and the peaks selected from the Rosetta magnetic field data also represent the $B_{0}$ values accurately. Differences between the two datasets could be caused by the peak selection pressure not requiring any estimate of the outgassing rate, therefore it is less sensitive to errors in the production rate estimates and differences between dark and illuminated parts of the orbit.

We also compared the results of our Rosetta pressure proxy to near-Earth solar wind pressure measurements extrapolated to the location of comet 67P. We found that near-Earth datasets (ACE, OMNI) give similar pressure results for all three propagation methods, while in late 2015 the propagated STEREO-A data differs strongly from both the near-Earth datasets and our Rosetta pressure proxy due to its great distance from both Earth and comet 67P.

In Figure 6 we compare our Rosetta pressure proxy calculated using the peak-selection method with OMNI data extrapolated by the magnetic lasso model (Dósa et al., 2018). Overall, our pressure proxy and the extrapolated OMNI pressure show good resemblance, especially in case of low solar activity. 

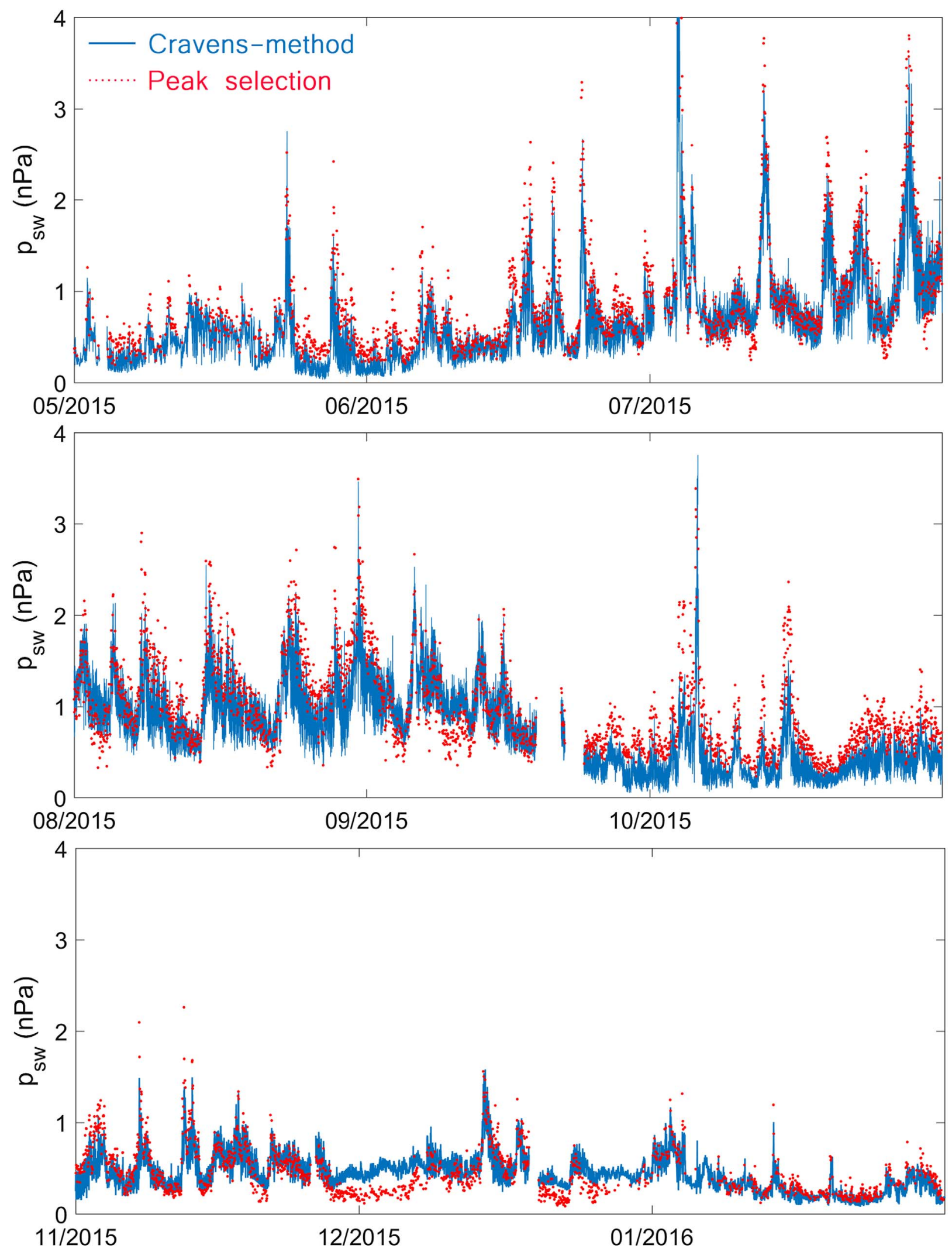

Fig. 5. Rosetta pressure proxy using peak-selection (red scatter points) compared to the Cravens neutral drag (blue line) model. Both of the illustrated results are averaged to remove the rapid pressure variations due to the high temporal resolution of the datasets. In most of October 2015 the spacecraft moved away from the comet and the diamagnetic cavity to its dayside excursion, therefore it was probably far away from the $B_{0}$ maximum, therefore the results, especially the Cravens-solution, probably underestimate the actual pressure this month. 

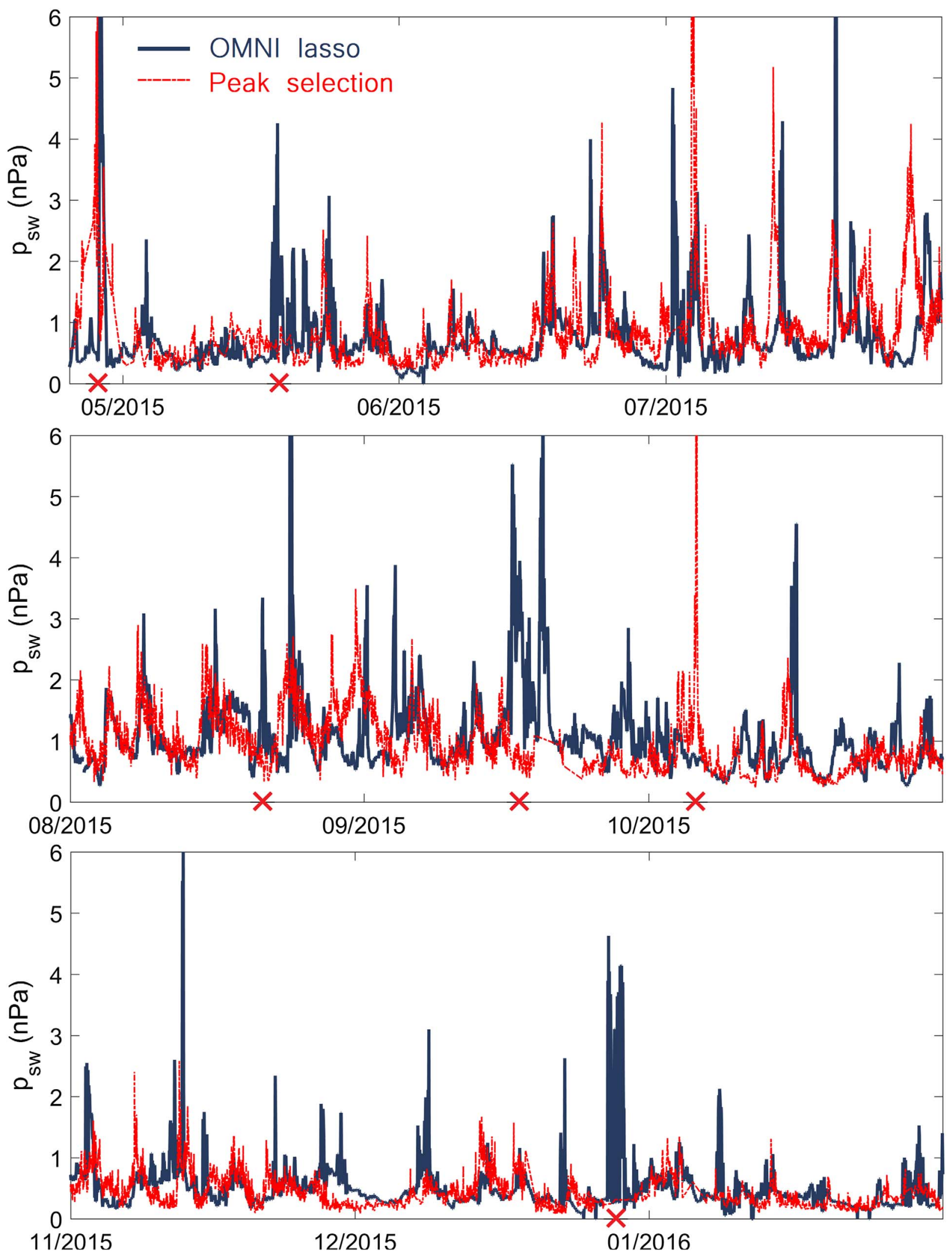

Fig. 6. Comparison of the Rosetta pressure proxy calculated using the peak selection method (red line) and the propagated OMNI lasso pressure (dark blue line) at comet 67P, between late April 2015 and January 2016. The pressure calculated using the peak selection method is averaged to remove the rapid solar wind fluctuations due to the temporal resolution of the data. The ICMEs hitting either Earth or comet $67 \mathrm{P}$ are marked by red crosses on the time axes. 

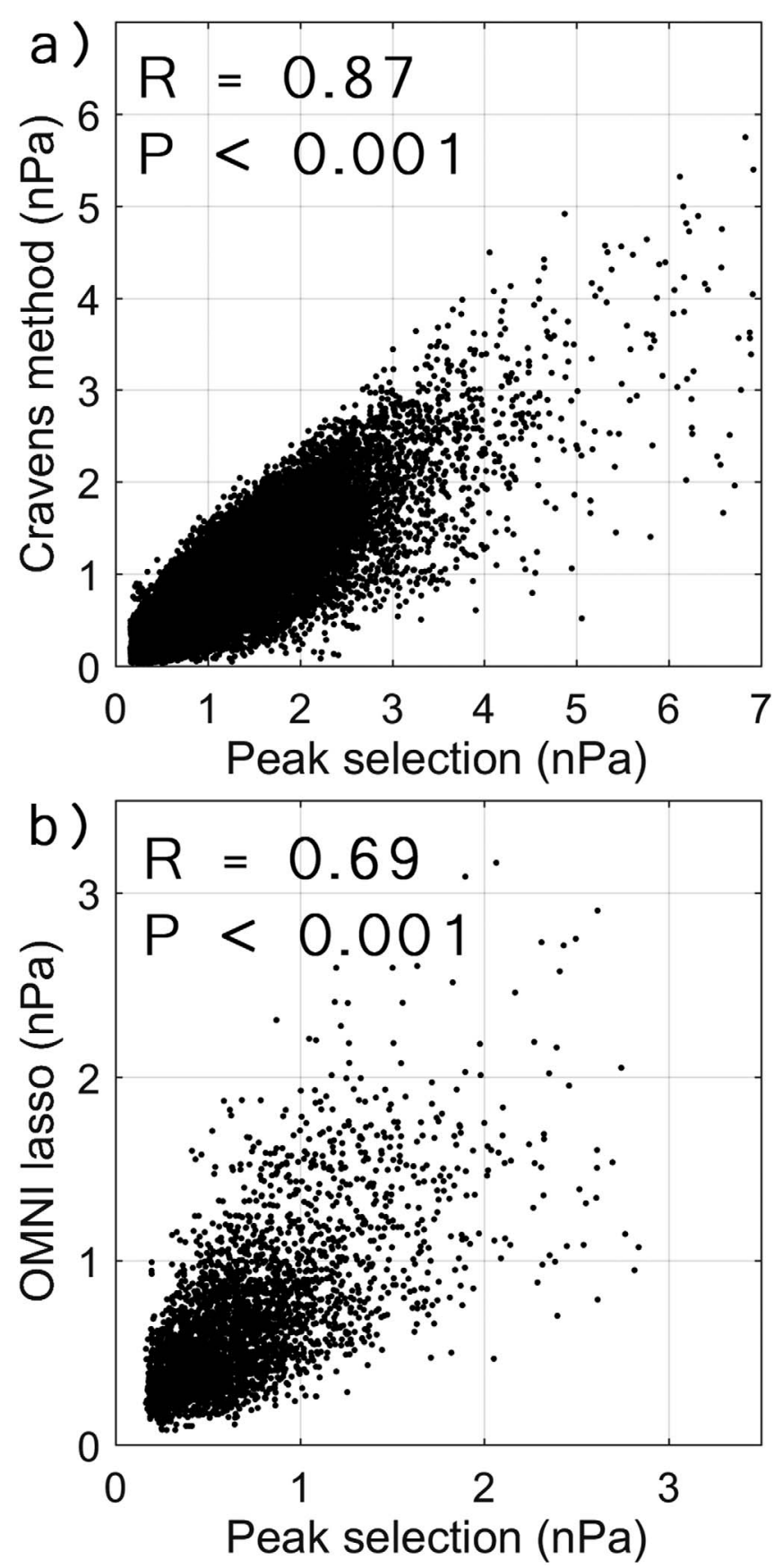

Fig. 7. Comparing the peak-selection method and the Cravens solution (a) below dynamic pressure values of $7 \mathrm{nPa}$. The two methods correlate well with a correlation coefficient $R=0.87$ and a $p$ value less than 0.001 . The plot shows that the Cravens solution tends to give lower estimates than the peak selection. In (b) we show the correlation between the peak-selection method and the OMNI lasso propagated solar wind dynamic pressure values. To calculate the correlation coefficient, we filtered out the transient events in the solar wind and got $R=0.69$ and $p<0.001$ showing a strong linear relationship between the two datasets.

There are a few cases, where significant difference can be observed, we discuss the probable causes later. The minimum solar wind pressure is around $0.2-0.4 \mathrm{nPa}$ in both the propagated data and the pressure proxy, showing a small increase near the comet's perihelion in mid-August up to $0.5-0.6 \mathrm{nPa}$.

The solar activity throughout the orbiting phase of the Rosetta mission was nearly at its peak. There were multiple coronal mass ejections (CMEs) (Edberg et al., 2016) and Corotating Interaction Regions (CIRs) in 2015 and early 2016 passing both near-Earth and near comet 67P, and there was rarely a week with continuously low activity. This, combined with the great distance between comet 67P and the Earth at the time, makes the solar wind pressure extrapolated from near-Earth to the comet less reliable. The arrival time of uninterrupted CIRs travelling at constant velocity can be predicted by the extrapolation methods with great confidence. For example, in Figure 4 we can see that in case of those CIRs which can uninterruptedly reach the comet after they moved past Earth, our proxy and the propagated solar wind pressure show strong similarity. In early to mid August 2015 there was no notable solar activity that could have significantly affected the quality of the model propagation of these CIRs from Earth. In general, our pressure proxy and the propagated OMNI dataset differ when interplanetary CMEs (ICMEs) are arriving either at comet $67 \mathrm{P}$ or at Earth; these transient events usually give false results in the propagation models. To account for these discrepancies between the two datasets, we searched for already reported transient events at the location of comet $67 \mathrm{P}$ in 2015, complemented with the measurements of spacecraft located near planet Mars (MAVEN) and the SOHO/ LASCO CME catalog (https://cdaw.gsfc.nasa.gov/CME_list/).

One of the prominent CME events that reached comet 67P around 6 October 2015 was described by Edberg et al. (2016). They reported that the ICME also was detected by the MAVEN and the MEX spacecraft at Mars, located relatively near 67P in early October 2015. The ICME impact was observed during the dayside excursion of the spacecraft, when Rosetta's distance from the nucleus was about $1500 \mathrm{~km}$. Despite Rosetta's great distance from the comet we can still observe a significant increase in the pressure proxy, but since Rosetta is also far away from the location of the maximum $B_{0}$ in the pile-up region, our proxy somewhat underestimates the maximum pressure value of the ICME. Similarly, in the first day of July 2015 the solar wind monitoring spacecraft at Mars reported the arrival of an ICME, which could also be responsible for the observed ICME hitting the comet on 3 July 2015 and causing our Rosetta pressure proxy to mismatch with the data propagated from near-Earth (Fig. 6). At the time of this event, comet 67P was located approximately $60^{\circ}$ off from the Sun-Mars line and more than 0.2 AU closer to the Sun than Mars. Due to the Earth's great distance from Mars and comet 67P, these CMEs missed Earth and are not present in the lasso dataset extrapolated from near Earth.

There are ICME impacts on both solar wind pressure datasets on 28 April 2015 separated only by a few hours (Fig. 6). At this time, comet 67P was located almost on the opposite side of the Sun relative to Earth (approximately $140^{\circ}$ off from the Sun-Earth line, see Fig. 1), thus these two peaks in the different datasets must mark two very different ICME events. The peak in the mSWiM data shows an ICME that only hit Earth and got falsely propagated all the way through the other side of the Sun to 67P, while according to the SOHO/LASCO catalog there were multiple CMEs heading 
towards the general direction of comet $67 \mathrm{P}$ between $23-25$ April 2015 that could have caused the observed impact in our Rosetta pressure proxy on 28 April 2015.

There were several other ICMEs hitting Earth while missing comet $67 \mathrm{P}$ through these few months. In these cases, there are peaks in the propagated near-Earth solar wind pressure with no corresponding peaks in our Rosetta pressure proxy. These events are marked with red crosses in Figure 6 along with the events when an ICME hit comet 67P while missing Earth.

Aside from the CMEs, there are also time periods where the two datasets differ with no clear ICME observations near-Earth or at comet 67P, for example in late July 2015 and from late August to the early days of September 2015 (Fig. 6). These discrepancies are probably the result of the (both spatially and temporally) changing properties of CIRs propagating past Earth towards comet $67 \mathrm{P}$.

After the exclusion of disturbances caused by transient events, we can conclude that the propagated pressure agrees well with the Rosetta pressure proxy (Fig. 7b).

The resulting Rosetta magnetic field-based pressure proxy is also far better at predicting the size of the diamagnetic cavity than the pressure values propagated from other spacecraft by solar wind propagation tools (Timar et al., 2017).

\section{Conclusion}

In this paper, we used the magnetic field measurements of the Rosetta spacecraft near the diamagnetic cavity of comet 67P/Churyumov-Gerasimenko to develop a proxy for the solar wind dynamic pressure around the comet for the time period between May 2015 and January 2016. We estimated the maximum of the magnetic field strength in the magnetic pile-up region of the induced cometary magnetosphere by two different methods: by inverting the solution of the neutral drag model of Cravens and by peak-selection. The two methods gave very similar results. This maximum is a measure of the solar wind dynamic pressure acting on the outside of the cometary magnetosphere. Thus, from the magnetic field measurements deep inside the magnetosphere we can deduce the pressure of the solar wind around the comet to create a Rosetta pressure proxy dataset for general use.

We validated our method using various solar wind propagation models applied to the measurements of different solar wind monitoring spacecraft. In case of low solar activity and in case of uninterrupted CIRs, the propagated solar wind pressure and our Rosetta pressure proxy show great resemblance. We usually found differences between the two datasets when transient events, like ICMEs hit one of the objects, because such events can bypass either Earth or the comet. To further validate our data, we investigated these transient events using SOHO and MAVEN data to account for the discrepancies between the propagations and our Rosetta pressure proxy. We also searched for already reported solar wind activities measured by Rosetta to compare them with our proxy. The results show good agreement.

The pressure proxy derived by this method can be used in investigations of other solar wind pressure sensitive plasma phenomena of comet 67P. It also makes the Rosetta Magnetometer a solar wind pressure monitor, data of which can be used as an input for space weather prediction models in other locations in the Solar System.

Acknowledgements. Rosetta is an ESA mission with contributions from its member states and NASA. We thank the Rosetta Mission Team, SGS, and RMOC for their outstanding efforts in making this mission possible. The authors acknowledge the ACE, STEREO, MAVEN and OMNI teams for providing data. We thank K.C. Hansen and B. Zieger for providing solar wind propagations from their Michigan Solar Wind Model. The SOHO LASCO CME catalog is generated and maintained at the CDAW Data Center by NASA and The Catholic University of America in cooperation with the Naval Research Laboratory. SOHO is a project of international cooperation between ESA and NASA. The work of ZN was supported by the János Bolyai Research Scholarship of the Hungarian Academy of Sciences. Part of the work was supported by Europlanet. Technical help was provided by Lajos Foldy and Klaudia Szabó. The editor thanks two anonymous referees for their assistance in evaluating this paper. This work was partially supported by OTKA.

\section{References}

Auster HU, Apathy I, Berghofer G, Fornacon KH, Remizov A, et al. 2015. The nonmagnetic nucleus of comet 67P/ChuryumovGerasimenko. Science 349: 6247, aaa5102. DOI: 10.1126/science. aaa5102.

Balsiger H, Altwegg K, Bochsler P, Eberhardt P, Fischer J, et al. 2007. Rosina - Rosetta orbiter spectrometer for ion and neutral analysis. Space Sci Rev 128: 745-801. DOI: 10.1007/s11214-006-8335-3.

Bieler A, Altwegg K, Balsiger H, Belthelier JJ, Calmonte U, et al. 2015. Comparison of 3D kinetic and hydrodynamic models to ROSINA-COPS measurements of the neutral coma of $67 \mathrm{P} /$ Churyumov-Gerasimenko. $A \& A$ 583: A7.

Boudouridis A, Zesta E, Lyons R, Anderson PC, Lummerzheim D. 2003. Effect of solar wind pressure pulses on the size and strength of the auroral oval. J Geophys Res 108: 8012. DOI: 10.1029/ 2002JA009373.

Clarke JT, Nichols J, Gérard JC, Grodent D, Hansen KC, et al. 2009. Response of Jupiter's and Saturn's auroral activity to the solar wind. J Geophys Res 114: A05210. DOI: 10.1029/2008JA013694.

Crary FJ, Clarke JT, Dougherty MK, Hanlon PG, Hansen KC, et al. 2005. Solar wind dynamic pressure and electric field as the main factors controlling Saturn's aurorae. Nature 433: 720-722. DOI: 10.1038/nature 03333 .

Cravens TE. 1986. The physics of the cometary contact surface. In: ESA SP-250: ESLAB Symposium on the Exploration of Halley's Comet, Battrick B, Rolfe EJ, Reinhard R (Eds.), ESA, Noordwijk, The Netherlands. p. 241.

Cravens TE. 1987. Theory and observations of cometary ionospheres. Adv Space Res 12: 147-158.

Cravens TE. 1991. Plasma processes in the inner coma. Int Astron Union Colloq 116: 121. DOI: 10.1017/S0252921100012884.

Crider DH, Vignes D, Krymskii AM, Breus TK, Ness NF, et al. 2003. A proxy for determining solar wind dynamic pressure at Mars using Mars Global Surveyor data. J Geophys Res 108(A12): 1461. DOI: 10.1029/2003JA009875.

Dósa M, Opitz A, Dálya Z, Szegö K. 2018. Magnetic lasso: an enhanced ballistic solar wind propagation method. Sol Phys 293: 127. DOI: $10.1007 / \mathrm{s} 11207-018-1340-3$. 
Dubinin E, Sauer K, Lundin R, Norberg O, Trotignon JG, et al. 1996. Plasma characteristics of the boundary layer in the Martian magnetosphere. J Geophys Res 101(A12): 27061-27075. DOI: 10.1029/96JA02021.

Edberg NJT, Eriksson AI, Odelstad E, Henri P, Lebreton JP, et al. 2015. Spatial distribution of low-energy plasma around comet 67P/CG from Rosetta measurements. Geophys Res Lett 42: 42634269. DOI: 10.1002/2015GL064233.

Edberg NJT, Alho M, André M, Andrews DJ, Behar E, et al. 2016. CME impact on comet $67 \mathrm{P} /$ Churyumov-Gerasimenko. MNRAS 462: S45-S56. DOI: 10.1093/mnras/stw2112.

Galand M, Héritier KL, Odelstad E, Henri P, Broiles TW, et al. 2016. Ionospheric plasma of comet 67P probed by Rosetta at $3 \mathrm{AU}$ from the Sun. MNRAS 462: S331-S351. DOI: 10.1093/mnras/stw2891.

Galeev AA. 1986. Theory and observations of solar wind/cometary plasma interaction processes. In: ESA Proceedings of the 20th ESLAB Symposium on the Exploration of Halley's Comet, 27-31 October 1986, Heidelberg, Germany, Vol. 1, pp. 3-17.

Gan L, Cravens TE. 1990. Electron energetics in the inner coma of comet Halley. J Geophys Res 95(A5): 6285-6303. DOI: 10.1029/ JA095iA05p06285.

Glassmeier KH, Boehnhardt H, Koschny D, Kührt E, Richter I. 2007a. The Rosetta mission: Flying towards the origin of the Solar System. Space Sci Rev 128: 1-21.

Glassmeier KH, Richter I, Diedrich A, Musmann G, Auster U, et al. 2007b. RPC-MAG the fluxgate magnetometer in the Rosetta Plasma Consortium. Space Sci Rev 128: 649-670.

Glassmeier KH. 2017. Interaction of the solar wind with comets: a Rosetta perspective. Philos Trans Ser A Math Phys Eng Sci 375 : 2097.

Goetz C, Koenders C, Hansen KC, Burch J, Carr C, et al. 2016. Structure and evolution of the diamagnetic cavity at comet $67 \mathrm{P} /$ Churyumov-Gerasimenko. MNRAS 462(Suppl_1): S459-S467. DOI: $10.1093 / \mathrm{mnras} / \mathrm{stw} 3148$.

Goetz C, Volwerk M, Richter I, Glassmeier KH. 2017. Evolution of the magnetic field at comet 67P/Churyumov-Gerasimenko. MNRAS 469(Suppl_2): S268-S275.

Hajra R, Henri P, Vallières X, Moré J, Gilet N, et al. 2018. Dynamic unmagnetized plasma in the diamagnetic cavity around comet 67P/Churyumov-Gerasimenko. MNRAS 475(3): 4140-4147. DOI: $10.1093 / \mathrm{mnras} /$ sty094.

Hansen C, Altwegg K, Berthelier JJ, Bieler A, Biver N, et al. 2016. Evolution of water production of $67 \mathrm{P} /$ Churyumov-Gerasimenko: an empirical model and a multi-instrument study. MNRAS 462: S491-S506. DOI: 10.1093/mnras/stw2413.

Hässig M, Altwegg K, Balsiger H, Bar-Nun A, Berthelier JJ, et al. 2015. Time variability and heterogeneity in the coma of $67 \mathrm{P} /$ Churyumov-Gerasimenko. Science 347: aaa0276.

Ip WH, Axford W. 1987. The formation of a magnetic-field-free cavity at comet Halley. Nature 325: 418. DOI: 10.1038/325418a0.

Luhmann JG, Elphic RC, Russell CT, Mihalov JD, Wolfe JH. 1980. Observations of large scale steady magnetic fields in the dayside Venus ionosphere. Geophys Res Lett 7: 917-920. DOI: 10.1029/ GL007i011p00917.

Madanian H, Cravens TE, Rahmati A, Goldstein B, Burch J, et al. 2016a. Suprathermal electrons near the nucleus of comet $67 \mathrm{P} /$ Churyumov-Gerasimenko at $3 \mathrm{AU}$ : Model comparisons with
Rosetta data. J Geophys Res 121: 5815-5836. DOI: 10.1002/ 2016JA022610.

Madanian H, Cravens TE, Burch J, Goldstein R, Rubin M, et al. 2016b. Plasma environment around comet 67P/ChuryumovGerasimenko at perihelion: Model comparison with Rosetta data. Astron J 153: 30. DOI: 10.3847/1538-3881/153/1/30.

Mandt KE, Eriksson A, Edberg NJT, Koenders C, Broiled T. 2016. RPC observation of the development and evolution of plasma interaction boundaries at 67P/Churyumov-Gerasimenko. MNRAS 462: S9-S22. DOI: 10.1093/mnras/stw1736.

Mendis DA, Houpis HLF, Marconi ML. 1985. The physics of comets. Fundam Cosm Phys 10(1-4): 380. p. 2. ISSN 0094-5846.

Nemeth Z, Burch J, Goetz C, Goldstein R, Henri P, et al. 2016. Charged particle signatures of the diamagnetic cavity of comet 67P/Churyumov-Gerasimenko. MNRAS 462(Suppl_1): S415S421. DOI: $10.1093 / \mathrm{mnras} / \mathrm{stw} 3028$.

Neubauer FM, Glassmeier KH, Pohl M, Raeder J, Acuna MH, et al. 1986. First results from the Giotto magnetometer experiment at comet Halley. Nature 321: 352.

Opitz A, Karrer R, Wurz P, Galvin AB, Bochsler P, et al. 2009. Temporal evolution of the solar wind bulk velocity at solar minimum by correlating the STEREO A and B plastic measurements. Sol Phys 256: 365. DOI: 10.1007/s11207-008-9304-7.

Richter I, Koenders C, Auster HU, Goetz C, et al. 2015. Observation of a new type of low-frequency waves at comet $67 \mathrm{P} / \mathrm{Churyumov}$ Gerasimenko. Ann Geophys 33: 1031-1036. DOI: 10.5194/ angeo-33-1031-2015.

Sibeck DG, Lopez RE, Roelof EC. 1991. Solar wind control of the magnetopause shape, location, and motion. J Geophys Res 96(A4): 5489-5495. DOI: 10.1029/90JA02464.

Timar A, Nemeth Z, Szego K, Dosa M, Opitz A, et al. 2017. Modelling the size of the very dynamic diamagnetic cavity of comet 67P/Churyumov-Gerasimenko. MNRAS 469(Suppl_2): S723-S730. DOI: 10.1093/mnras/stx2628.

Vennerstrom S, Olsen N, Purucker M, Acuña MH, Cain JC. 2003. The magnetic field in the pile-up region at Mars, and its variation with the solar wind. Geophys Res Lett 30: 1369. DOI: 10.1029/ 2003 GL016883.

Vigren E, Altwegg K, Edberg NJT, Eriksson AI, Galand M, et al. 2016. Model-observation comparisons of electron number densities in the coma of 67P/Churyumov-Gerasimenko during 2015 January. Astron J 152: 59 (8 pp). DOI: 10.3847/0004-6256/152/3/59.

Vigren E, Eriksson AI. 2017. A 1D model of radial ion motion interrupted by ion-neutral interactions in a cometary coma. Astron $J$ 153: 4.

Wedlund CS, Alho M, Gronoff G, Kallio E, Gunell H, et al. 2017. Hybrid modelling of cometary plasma environments: I. Impact of photoionisation, charge exchange, and electron ionisation on bow shock and cometopause at 67P/Churyumov-Gerasimenko. $A \& A$ 604: A73. DOI: 10.1051/0004-6361/201730514.

$\mathrm{Wu} \mathrm{ZJ}$. 1987. Calculation of the shape of the contact surface at comet Halley. In: ESA proceedings of the International Symposium on the Diversity and Similarity of Comets 6-9 April 1987, Brussels, Belgium, 69-73.

Zieger B, Hansen KC. 2008. Statistical validation of a solar wind propagation model from 1 to $10 \mathrm{AU}$. J Geophys Res, 113, A08107. DOI: $10.1029 / 2008 J A 013046$.

Cite this article as: Timar A, Nemeth Z, Szego K, Dósa M, Opitz A, et al. 2019. Estimating the solar wind pressure at comet 67P from Rosetta magnetic field measurements. J. Space Weather Space Clim. 9, A3. 